\section{Graves' disease: The thyroid in 'overdrive'}

\author{
MARGARET REICH \\ Senior Staff Editor
}

As the most common type of a malfunctioning, overactive thyroid gland, Graves' disease strikes only about 20 to 200 people per 100,000 . (First Lady Barbara Bush is among this select population.) Onset most commonly occurs in adults between the ages of 20 and 40 . Women are seven to ten times more likely than men to have this disorder. However, when Graves' disease strikes men, it happens at a later age and tends to be more severe. Heredity seems to play some part. Studies have found Graves' disease occurs more often in identical twins than in the rest of the population.

\section{Thyroid anatomy}

The butterfly-shaped thyroid gland is located just above the windpipe (trachea) and just below the voicebox (larynx) in the throat. A thin strand of hormone tissue (isthmus) joins the two lobes that make up the gland. These lobes are located on either side of the windpipe.

Two pea-sized parathyroid glands can be found next to these two lobes. Parathyroids help regulate calcium levels in the body.

\section{The precision team}

As a key player in maintaining the body's metabolism, the thyroid requires an outside source, iodine, to help produce its hormones - thyroxine $\left(T_{4}\right)$ and triiodothyronine $\left(\mathrm{T}_{3}\right)$. (The third thyroid hormone, calcitonin, is regulated by the amount of calcium found in the blood. As such, it works with the parathyroid hormone to control calcium levels in the body.)

The thyroid-stimulating hormone (TSH) controls the amount of $\mathrm{T}_{4}$ and $\mathrm{T}_{3}$. In turn, $\mathrm{TSH}$ is secreted by the pituitary gland, which is connected to the hypothalamus in the brain. The hypothalamus wears many hats. As the "command central" of the sympathetic nervous system, it initiates the "flight or fight" response, and controls body temperature and mood, to name but a few functions.

The slightest altercation disrupts the precise teamwork necessary for a smooth-running thyroid.

\section{The body in 'overdrive'}

In a hyperthyroid gland, even though normal TSH levels may exist, the gland continues to produce excess $T_{4}$ and $T_{3}$ amounts. With this added "fuel," the body's mental and physical processes speed up. Anxiety increases substantially. Despite a ravenous appetite, weight loss usually occurs. Other common manifestations include rapid heart beat; insomnia; muscle weakness; sensitivity to heat and cold; increased sweating; warm moist skin; excessive thirst and urination; menstrual irregularities; and an inability to concentrate. Sometimes, slight, but uncontrollable finger and tongue movements may occur.

An enlarged gland (goiter), often producing a noticeable swelling in the neck; bulging eyeballs with a staring appearance (exophthalmos); and swelling of the skin on the front of the legs with a dry, waxy appearance (myxedema) are classic Graves' disease characteristics. However, in reality, such classic symptoms don't always occur at once, especially in the elderly.

\section{Searching for the cause \\ Immune system}

What triggers the hormone overproduction, responsible for putting the body in "overdrive,"remains uncertain. However, medical experts believe this disorder can be traced to a malfunction in the immune system, which involves the antibodies. Found in white blood cells, antibodies are the "soldiers" of the immune system. They stand ready to attack any foreign invaders that might precipitate infection, for example. In Graves' disease, something goes awry; the antibodies latch onto normal thyroid tissue.

Explains Arthur Levine, DO, staff endocrinologist and director of nuclear medicine at Bi-County Community Hospital in Warren, Michigan: "Stress, whether physical or psychological, sets off a preexisting condition in the thyroid so that the body 
starts developing antibodies against the thyroid. This causes an inflammatory process in the thyroid, and excessive amounts of hormone are secreted.'

In fact, onset of Graves' disease frequently occurs during adolescence, pregnancy, and menopause, all times of great hormonal change.

\section{Diet}

In rare instances, too much dietary iodine can cause iodine-induced hyperthyroidism in someone susceptible to Graves' disease. But the average American diet poses no real threat in this instance.

Results from the Total Diet Study, 1982-1986, conducted by the Food and Drug Administration, found overall consumption of iodine decreased $24 \%$ during this 4 -year period. Although women, aged 25 to 30 years, consumed an average of $196.2 \%$ of the Recommended Dietary Allowances (RDA) of iodine in 1986, Dr Levine believes it would take massive amounts of daily iodine consumption to increase the hyperthyroid risk.

\section{Diagnosing the condition}

Even though family history and a physical examination may provide clues to the presence of Graves' disease, laboratory tests confirm the diagnosis. Fortunately, these painless tests are usually done on an outpatient basis.

\section{Blood tests}

As the primary diagnostic tool, blood tests can measure $\mathrm{T}_{4}, \mathrm{~T}_{3}$, and TSH levels. No fasting is required prior to testing. Those with a needle phobia or who faint at the sight of blood are probably the only persons who may find this procedure uncomfortable.
If blood test results prove inconclusive, your physician will probably order a thyroid scan and radioactive iodine uptake test.

\section{Radionuclide scan and}

radioactive iodine uptake test

One way to gauge the thyroid's performance is to measure the rate at which iodine accumulates in the

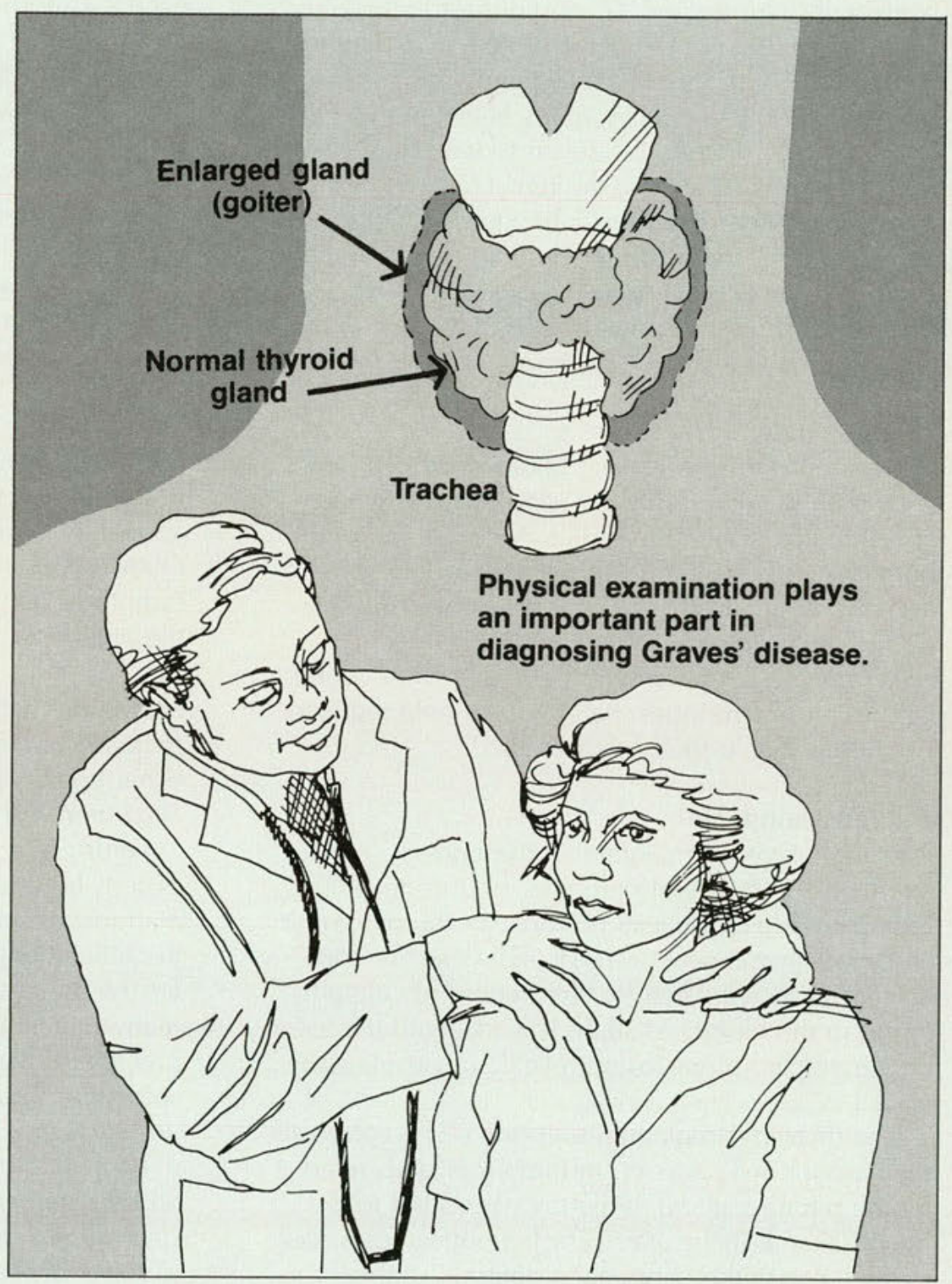


gland. The radionuclide scan with the radioactive iodine uptake test provides the means for this measurement.

But before any radioactive iodine is swallowed or thyroid pictures are taken, certain pretesting precautions should be heeded. While your physician will provide you with a complete list of dos and don'ts, here's a sneak preview. Avoid fish and any other food or medication (such as some asthma preparations) with iodine for at least one day; and fast overnight.

The actual procedure causes no pain. After swallowing the capsule or liquid containing the radioisotope material, you find a specially designed camera placed in front of your neck that will "photograph" the thyroid. Once the radioisotope enters the body, more than one scan is performed at different intervals. The amount of radioisotope taken up by the thyroid reveals whether or not hyperthyroidism exists. (This diagnostic procedure can also detect hypothyroidism and thyroid cancer.)

While the thyroid absorbs most of the radioactive material, trace elements may be absorbed by other tissues. The excess radioisotope is excreted in the urine in approximately 1 week.

The test is safe." The [radioactive] equivalent is probably less than having a chest $\mathrm{x}$-ray. Any need [to have the scan] outweighs the minimal risk," declares Dr Levine.

While pregnant women probably could undergo this test, Dr Levine notes that for safety sake, thyroid scans are not performed on these patients. However, children do undergo this procedure when needed.

\section{Choosing a treatment}

Armed with a Graves' disease diagnosis, your physician determines which therapy-radioactive iodine treatment, antithyroid hormone supplements, or surgery -is appropriate. As might be expected, individual factors - age, sex, severity of the disorder, other medical problems, and last (but not least) patient preference-play an important part in choosing an appropriate therapy.

No "best" treatment exists; each has its own advantages and disadvantages as well as advocates among physicians. Radioactive iodine therapy seems the current favorite. Yet, none of these therapies offers a real cure. In fact, the possibility for hypothyroidism, an underactive thyroid, exists with each of these approaches.

\section{Radioactive iodine therapy}

Similar to the iodine uptake test, this therapy requires drinking or swallowing a clear liquid containing a higher dosage of radioactive iodine. This increased dose damages or destroys the overactive thyroid cells that secrete $T_{4}$ and $T_{3}$. (It does not increase the chance of thyroid cancer.) With that, the gland decreases in size, and normal thyroid function usually resumes. Most patients require only one dose for the treatment to work.

Pregnant women cannot receive this treatment. However, Dr Levine says he has safely administered radioactive iodine therapy to children as young as 6 years old.

\section{Special precautions}

For a few days following treatment, you will be instructed to avoid coming in contact with pregnant women and children. Even your spouse will have to sleep alone for a while.

Because your body will emit radiation for about 3 days, wash your clothing and dishes separately from those of other household members. Also, because excreted urine will contain trace elements of radioactive material, Dr Levine suggests flushing the toilet three times. Finally, female patients should not become pregnant for at least 6 months to 1 year following treatment.

\section{Antithyroid hormone supplements}

Just as their name suggests, these pills suppress the production of hormones. Long-term treatment ( 1 to 2 years) is usually necessary to bring the thyroid under control. Unlike radioactive iodine treatment, antithyroid supplements may be administered to pregnant women under close physician supervision.

Weight gain, normal heart beat, and less anxiety will be noticeable within about 2 weeks of therapy. Like most medication, the hormone supplements may cause side effects, such as nausea and inability to taste food. Just as with radioactive iodine treatment, 
everyone will not respond to antithyroid supplements. Usually radioactive iodine therapy serves as the next option in this case.

\section{Surgery}

Although surgery (thyroidectomy) is not used as often as the other two therapies, it may be performed if a very large goiter or nodule exists. To prevent thyroid storm, a rare, but potentially serious condition, antithyroid medication is administered to stabilize the thyroid's activity. The activity of the gland generally stabilizes after approximately 1 to 3 months of antithyroid therapy. Surgery can then be scheduled.

Occasionally, Graves' disease will resolve without any intervention. Yet, left unchecked, it can contribute to calcium loss in bones (osteoporosis). Actually, the emotional distress and inability to concentrate, rather than physical complications, motivate most persons to seek medical help.

\section{Staying healthy}

Follow-up examinations are paramount for maintaining a healthy thyroid. How often you must see your physician depends, generally, on the type of therapy initially prescribed. Blood tests are usually done to monitor hormone levels. Should hypothyroidism ensue, appropriate treatment can be prescribed during this time, too.

Seeing your physician regularly, eating a proper diet, and avoiding strenuous activity go a long way in the campaign for healthy living.
(All material in the Patient Health Guide is reviewed by JAOA physician referees.)

\section{References:}

The American Medical Association Encyclopedia of Medicine. Edited by Charles B. Clayman. Random House, New York, 1989.

The American Medical Association Family Guide. Edited by Jeffrey R.M. Kunz and Asher J. Finkel. Random House, New York, 1987.

Clinical management of patients with hyperthyroidism. By David S. Cooper and E. Chester Ridgway. Medical Clinics of North America, vol 69, pp 953-968, Sept 1985.

The Columbia University College of Physicians and Surgeons Complete Home Medical Guide. Edited by Donald F. Tapley, et al. Crown Publishers, Inc, New York, 1985.

Current Medical Diagnosis \& Treatment. Edited by Steven A. Schroeder, Marcus A. Krupp, and Lawrence M. Tierney, Jr. Appleton \& Lange, Norwalk, Conn, 1988.

Getting the most from thyroid tests. By Jerome M. Hershman, Paul W. Ladenson, and Bernadine Z. Paulshock. Patient Care, vol 23, pp 86-106, Apr 30, 1989.

Hyperthyroidism, antithyroid drug preparation, and iodine 131 therapy (letter). By Martin L. Nusynowitz. The Journal of the American Medical Association, vol 252, pp 1761-1762, Oct 5, 1984.

Hyperthyroidism: Causes, clinical features, and diagnosis. By Stephen Spaulding and Howard Lippes. Medical Clinics of North America, vol 69 , pp 937-951, Sept 1985.

Hyperthyroidism in The Merck Manual of Diagnosis and Therapy. Edited by Robert Berkow, et al. Ed 15. Merck Sharp \& Dohme Research Laboratories, Rahway, NJ, 1987

Nutritional elements in US diets: Results from the Total Diet Study, 1982 to 1986. Journal of the American Dietetic Association, vol 89, pp 659-662, May 1989.

Textbook of Endocrinology. Edited by Jean D. Wilson and Daniel W. Foster. Ed 5. WB Saunders Co, Philadelphia, 1985.

\section{Look for future Patient Health Guides discussing Lyme disease and Alzheimer's disease.}

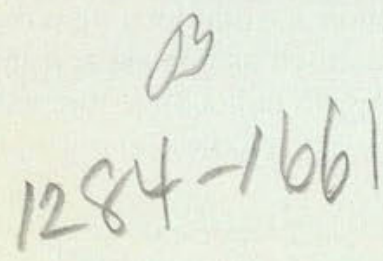




\section{Coming soon...}

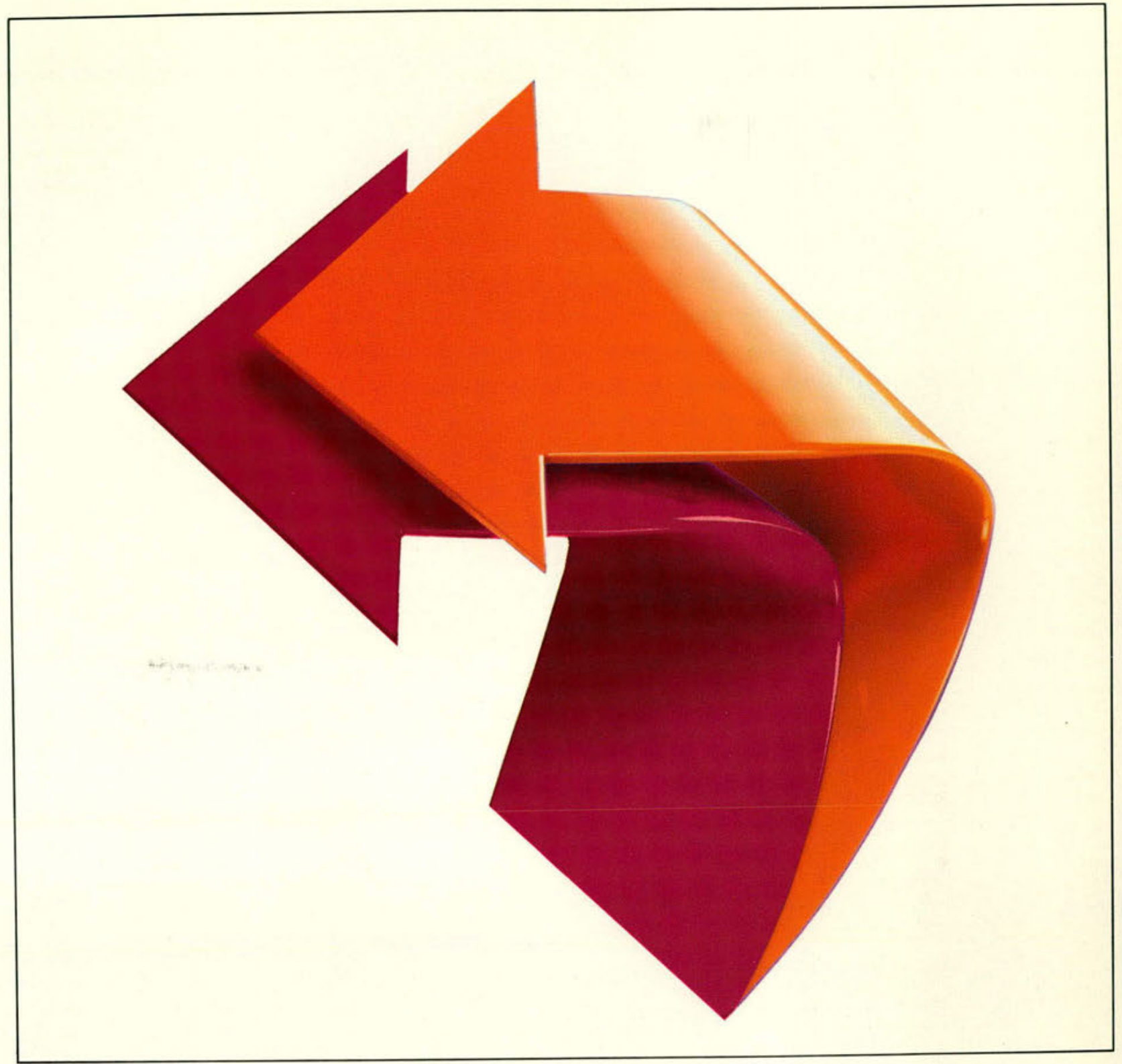

\section{An ideal new companion for}

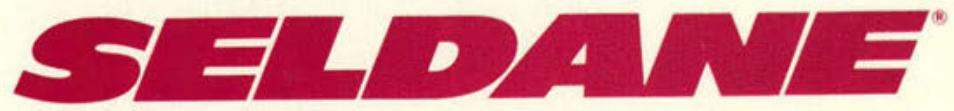

(terfenadine)

\section{Merrell Dow}




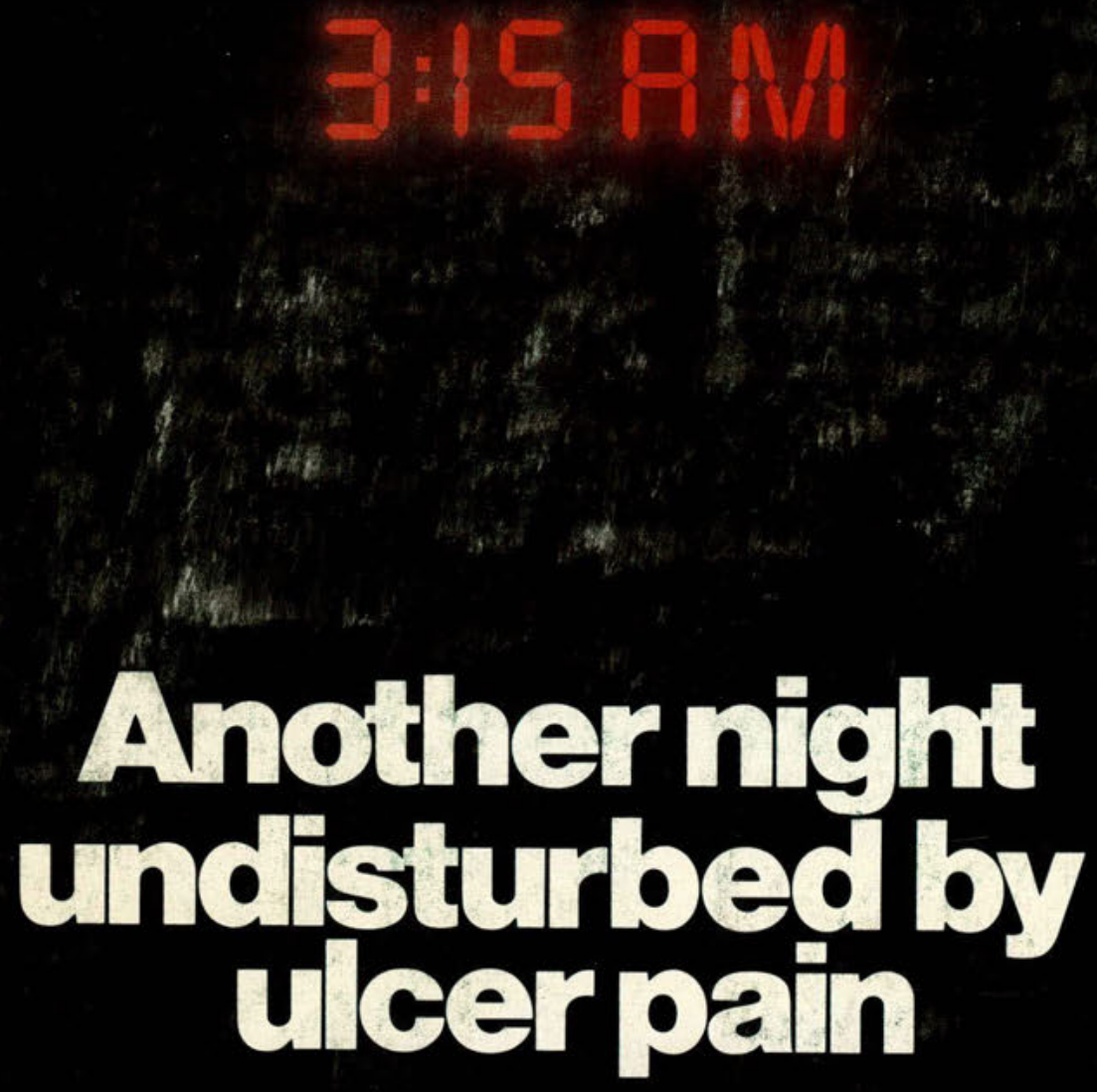

The Promise ... 'Tagamet' helps ulcer patients feel better fast.

The Proof ... $80 \%$ of patients with duodenal ulcer, and $77 \%$ with gastric ulcer...even smokers, get pain relief with their first dose of 'Tagamet' $800 \mathrm{mg}$. h.s. That's effectiveness. That's fast relief.

For fast relief, rapid healing, effective prevention of duodenal ulcer recurrence, the longest record of safety and unmatched value, there's nothing like...
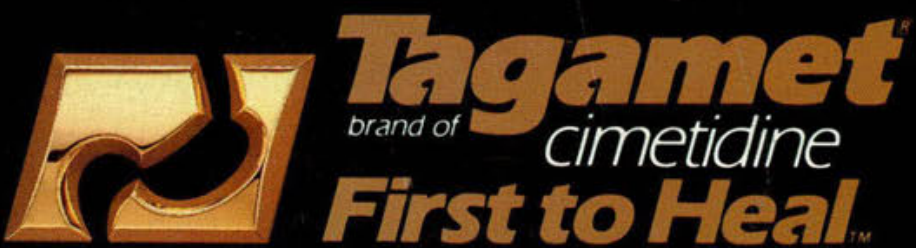

First available, First in experience... First to Heal ${ }_{T M}$
TAGAMET' (brand of clmetidline)

See complete prescribling information In SKEF LAB CO. Itera ture or PDR. The following is a brief summary.

Contraindications: Tagamet' is contraindicated for patients known to have hypersensitivity to the product

Precautions Rare instances of cardiac arrhythmias and hypotensio have been reported following the rapid administration of 'Tagamet' HC. (brand of cimetidine hydrochioride/ injection by intravenous bolus. Symptomatic response to Tagamet' therapy does not preclude the pres
ence of a gastric malignancy There have been rare reports of transien healing of gastric ulcers despite subsequently documented mallignancy. Reversible confusional states have been observed on occasion, predom nantly in severely ill patients.

Tagamet' has been reported to reduce the hepatic metabolism of warlarin-type anticoagulants, phenytoin, propranolol, chlordiazepoxide

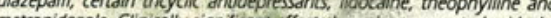
metronidazole. Clinically significant effects have been reported with the wartarin anticoagulants, therefore, close monicoring of prothrombin time is recommended, and adjustment of the anticoagylant dose may be necessary when tagamet is administered concombantly. Interaction with phenytoin, lidocaine and theophylline has also been reported to produce adverse clinical effects.

However, a crossover study in healthy subjects receiving either Tagamet $300 \mathrm{mg}$. q. id. or $800 \mathrm{mg}$. h.s. concomitantly with a $300 \mathrm{mg}$. b.i.d. dosage of theophylline (Theo-Dur, Key Pharmaceuticals, Inc.) demonstrated less alteration in steady-state theophylline peak serum levels with the 800 $m g$. h.s. regimen, particularly in subjects aged 54 years and older. Dato beyond ten days are not avarlable. (Note: All patients receiving theophy line should be monitored appropriately, regardless of concomitant drug therapy.

In a 24 month toxicity study in rats, at dose levels approximately 8 to 48 times the fecommended human dose, benign Leydig cell tumors were seen. These were common in both the treated and control groups, and the incidence became significantly higher only in the aged rats receiving Tagamet

A weak antiandrogenic effect has been demonstrated in animals in hu man studies, 'Tagamet' has been shown to have no effect on spermatogenesis, sperm count, motility, morphology orin vitro fertilizing capacity. Pregnancy Category 8: Reproduction studies have been performed in Tats, rabovits and mice at doses up to 40 times the normal human dose and have revealed no evidence of impaired fertility or harm to the fetus due to Tagamet. There are, however, no adequate and well-controlled studies in pregnant women, Because animal reproductive studies are not at ways predictive of human response, this drug should be used during pregnancy only if clearly needed.

Lack of experience to date precludes recommending 'Tagamet' for use in children under 16 unless anticipated benefits outweigh potential risks generally, nursing should not be undertaken by patients taking the drug since cimetidine is secreted in human milk.

Adverse Reactions: Diarrhea, dizziness, somnolence, headache. Re versible confusional states (e.g. mental confusion, agitation, psychosis, depression, anxiety, hallucinations, disorientation), predominantly in se verely ill patients, have been reported. Reversible impotence in patients

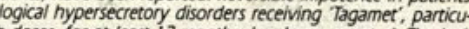
y in high doses, for at least 12 months, has been reported. The incidence of impotence in large-scale surveillance studies at regular doses has not exceeded that commonly reported in the general population. Gyne comastia has been reported in patients treated for one month or longer. Decreased white blood cell counts in Tagamet'-treated patients (approximately I per 100,000 patients), including agranulocytosis (approximately 3 per million patients), have been reported, including a few reports of reserious concomitant illnesses and received drugs and or who had known to produce neutropenia throment million reported increased serum transaminase has been a mia have asso been verse hepatic effects, cholestatic or mixed cholestaticheparensible aderare have been static feaves static features, severe parenchymal injury is considered highly unlikely. A ing 'Tagamer' has been reported Increased plasma creatinine has becen. ing Tagamer has been reported. Increased plasma creatinine has been reatitis and alfergic reactions, including anaphylaris and retention, pancreattis and allergic reactions, including anaphylaxis and hypersensitivity and A V have been reponted. Rare cases of bradycardia, tachycardia and A.V heart block have been reported with Hirreceptor antagonists. Reversibie anthralgia, myalgia and exacerbation of joint symptoms in patients with preexisting arthritis have been reported rarely. Rare cases of tablished. Mild rash and, very rarely, cases of severe generalized skin reactions (e.g. Stevens.Johnson syndrome, epidermal necrolysis, erythema ditione, exfoliative dermatits and generalized extoliative erychroderma) have been reported with $\mathrm{H}_{2}$-receptor antagonists. Reversible alopecia has been reported very rarely.

How Supplled: Tablets: $200 \mathrm{mg}$. tablets in bottles of 100; $300 \mathrm{mg}$. tab lets in bottles of 100 and Single Unit Packages of 100 (intended for institutional use onlyl: $400 \mathrm{mg}$. tablets in bottles of 60 and Single Unit Packages of 100 (intended for institutional use only), and $800 \mathrm{mg}$. Tittab tablets in bottles of 30 and Single Unit Packages of 100 lintended for institutional use only.

Llquid: $300 \mathrm{mg} .15 \mathrm{~mL}$, in $8 \mathrm{fl} .0 \mathrm{z}$. (237 mL.) amber glass bottles and in single-rose units $1300 \mathrm{mg} .15 \mathrm{~mL}$.), in packages of 10 (intended for institu. tional use only.

In/ection:

Vials: $300 \mathrm{mg} .12 \mathrm{~mL}$, in single-dose vials, in packages of 10 and 30 , and in $8 \mathrm{~mL}$. multiple-dose vials, in packages of 10 and 25 .

Prefilled Syringest $300 \mathrm{mg} / 2 \mathrm{~mL}$. in single-dose prefilled disposable syringes.

Single-Dose Prem/xed Plastic Contalners: $300 \mathrm{mg}$ in $50 \mathrm{~mL}$ of $0.9 \%$ sodium Chloride in singledose plastic containers in packages of 4 units. No preservative has been added.

Exposure of the premixed product to excessive heat should be avoided. It is recommended the product be stored at controlled room temperature. Brief exposure up to $40^{\circ} \mathrm{C}$ does not adversely affect the premixed prod

ADD-Vantage" Vlals: $300 \mathrm{mg} / 2 \mathrm{~mL}$. in single-dose ADD-Vantage" Vials, in packages of 25 .

'Tagamet' HCI (brand of cimetidine hydrochloride) Injection premixed in single-dose plastic containers is manulactured for SK\&F Lab CO. by Baxter Healthcare Corporation, Deerfiela. IL 60015.

'ADD-Vantage' is a urademark or Anoort Latoratories.

BRS-TG:L78B

Date of issuance Aug. 1988

SKEF LAB CO., Cidra, PR. 00639

o SK\&FLab CO., 1989 